\title{
Time-Dependent Density-Functional Theory in the Linear-Response Regime
}

\author{
Tai Kai $\mathrm{Ng}^{(\mathrm{a})}$ and K. S. Singwi \\ Department of Physics and Astronomy, Northwestern University, Evanston, Illinois 60201 \\ (Received 22 June 1987)
}

\begin{abstract}
The possibility of formulating a density-functional theory for time-dependent systems is studied in the linear-response regime. Two results are obtained: (i) The work of Runge and Gross is generalized to a finite time interval with an arbitrary form of the external potential; and (ii) there exists no zero in the mapping $n(\mathbf{r}, \omega)=\int d^{3} r^{\prime} \chi\left(\mathbf{r}, \mathbf{r}^{\prime} ; \omega\right) V\left(\mathbf{r}^{\prime}, \omega\right)$ for all $\omega$ under a very general thermodynamic condition.
\end{abstract}

PACS numbers: $31.10 .+\mathrm{z}, 03.65 .-\mathrm{w}, 71.10 .+\mathrm{x}$

The many successful applications of the densityfunctional theory to stationary systems have recently stimulated much effort to generalize the theory to timedependent systems. For a discussion of scattering and excitation phenomena, such a generalization is necessary. As in the stationary case, a fundamental existence theorem like that of Hohenberg and Kohn ${ }^{1}$ is necessary for the formal justification of the time-dependent density-functional theory. In this Letter, we study the possibility of having such an existence theorem in the linear-response regime. More precisely, the problem can be stated as follows: For a system of interacting electrons moving in a static external potential $v(\mathbf{r})$, the Hohenberg-Kohn ${ }^{1,2}$ theorem states that there exists a one-to-one correspondence between the external potential $v(\mathbf{r})$ and the ground-state electron density $n(\mathbf{r})$, when the ground state is nondegenerate. The question is, can this theorem be generalized to the case when the external potential is time dependent? ${ }^{2}$ In this connection the most interesting work is perhaps that of Runge and Gross $^{3}$ and its extension. ${ }^{4-6}$ Runge and Gross proved that for external potentials which can be expanded into a Taylor series with respect to the time coordinate around time $t=t_{0}$, where $t_{0}$ is the time the external timedependent potential starts to function, and if $k$ is the minimal nonnegative integer such that

$$
\frac{\partial^{k}}{\partial t^{k}}\left[v_{1}(\mathbf{r}, t)-v_{2}(\mathbf{r}, t)\right]_{t=t_{0}} \neq C(t)
$$

where $C(t)$ is a function independent of $\mathbf{r}$ and $v_{1}$ and $v_{2}$ are two different external time-dependent potentials, then

$$
\frac{\partial^{k+2}}{\partial t^{k+2}}\left[n_{1}(\mathbf{r}, t)-n_{2}(\mathbf{r}, t)\right]_{t=t_{0}} \neq 0
$$

under appropriate boundary conditions. ${ }^{6}$

In this Letter we shall examine this problem in the linear-response regime, when the change of the system induced by the external time-dependent potential is small. We shall consider two different situations: (i) Suppose we have a stationary state described by a density matrix $\rho$ at time $t<t_{0}$, and a time-dependent external potential of finite strength is being switched on at $t=t_{0}$.
For a short period of time $t-t_{0} \ll V_{0}^{-1}$, where $V_{0}$ measures the magnitude of the external potential, the change in the density matrix would be small, and the problem can be treated in a linear-response theory. (ii) The external potential is small.

Time-dependent density-functional theory in the short-time domain.-Consider a system of interacting electrons in a stationary state at time $t<t_{0}$. The stationary state can be a quantum state or can be described by a density matrix $\rho$ for a system in thermal equilibrium. Suppose we turn on a time-dependent external potential $v(\mathbf{r}, t)$ at time $t=t_{0}$. As long as $v(\mathbf{r}, t)$ is finite, there exists a region in time $t-t_{0}<\tau$ such that the induced change in the state is small and can be expanded in a series in $v(\mathbf{r}, t)\left(t-t_{0}\right)$. Obviously, for this expansion to converge, the period $\tau$ should be chosen such that $V_{0} \tau \ll 1$, where $V_{0}$ is a measure of the magnitude of the external potential at $t<\tau$.

The induced density fluctuation $n(\mathbf{r}, t)$ at this time interval can be obtained in this case to first order in $V_{0}\left(t-t_{0}\right)$ via linear-response theory, where

$$
n(\mathbf{r}, t) \sim \int d^{3} r^{\prime} \int_{t_{0}}^{t} d t^{\prime} \chi\left(\mathbf{r}, t ; \mathbf{r}^{\prime}, t^{\prime}\right) v\left(\mathbf{r}^{\prime}, t^{\prime}\right),
$$

$\chi\left(\mathbf{r}, t ; \mathbf{r}^{\prime}, t^{\prime}\right)$ being the usual density-density response function. ${ }^{7}$ To proceed further, we consider the short-time behavior of the response function.

The $n$th moment of the density-density response function is defined as

$$
\left\langle\omega^{n}\right\rangle_{\mathbf{r}, \mathbf{r}^{\prime}}=\int_{-\infty}^{+\infty} \chi\left(\mathbf{r}, \mathbf{r}^{\prime} ; \omega\right) \omega^{n} d \omega
$$

where

$$
\chi\left(\mathbf{r}, \mathbf{r}^{\prime} ; \omega\right)=\int_{0}^{\infty} \chi\left(\mathbf{r}, \mathbf{r}^{\prime} ; t\right) e^{(i \omega-\eta) t} d t .
$$

If all moments of the response function are finite, then for small enough time $t-t_{0}, \chi\left(\mathbf{r}, \mathbf{r}^{\prime} ; t\right)$ can be expanded in a Taylor series in $t-t_{0}$, where

$$
\chi\left(\mathbf{r}, \mathbf{r}^{\prime} ; t\right)=\frac{1}{2 \pi} \sum_{n=0}^{\infty}\left\langle\omega^{n}\right\rangle_{\mathbf{r}, \mathbf{r}^{\prime}} \frac{(-i t)^{n}}{n !} .
$$

The moments $\left\langle\omega^{n}\right\rangle_{\mathbf{r}, \mathbf{r}^{\prime}}$ are given by exact sum rules, ${ }^{7}$ 
where

$$
\left\langle\omega^{n}\right\rangle_{\mathbf{r}, \mathbf{r}^{\prime}}=2 \pi i\left\langle\left[[\ldots[n(\mathbf{r}), H] \ldots, H]^{n}, n\left(\mathbf{r}^{\prime}\right)\right]\right\rangle
$$

for $n \geq 1$, and

$$
\left\langle\omega^{0}\right\rangle_{\mathbf{r}, \mathbf{r}^{\prime}}=2 \pi i\left\langle\left[n(\mathbf{r}), n\left(\mathbf{r}^{\prime}\right)\right]\right\rangle=0 .
$$

The angular brackets on the right-hand side of Eqs. (7) denote the expectation values of the quantities inside the brackets over the stationary quantum state at $t=t_{0}$, or the ensemble average. Notice that we have set $\hbar=1$ everywhere in our derivation.

With Eqs. (3), (6), and (7), we find that for small enough time $t-t_{0}$,

$$
\left.n(\mathbf{r}, t) \sim(-i / 2 \pi) \int d^{3} r^{\prime}\left\langle\omega^{1}\right\rangle_{\mathbf{r}, \mathbf{r}^{\prime}} \int_{t_{0}}^{t}\left(t-t^{\prime}\right) v\left(\mathbf{r}^{\prime}, t^{\prime}\right)\right) d t^{\prime}
$$

It is easy to show that ${ }^{7}$

$$
\left\langle\omega^{1}\right\rangle_{\mathbf{r}, \mathbf{r}^{\prime}}=(2 \pi i / m)\left(\nabla_{\mathbf{r}} \cdot \nabla_{\mathbf{r}^{\prime}}\right)\left[\langle n(\mathbf{r})\rangle_{\delta}\left(\mathbf{r}-\mathbf{r}^{\prime}\right)\right],
$$

where $\langle n(\mathbf{r})\rangle$ is the static density distribution in the stationary state. Therefore,

$$
n(\mathbf{r}, \mathbf{t}) \sim(-1 / m) \nabla \cdot\left[\langle n(\mathbf{r})\rangle \int_{t_{0}}^{t} d t^{\prime}\left(t-t^{\prime}\right) \nabla v\left(\mathbf{r}, \mathbf{t}^{\prime}\right)\right]
$$

for short enough time interval.

Let $v_{1}(\mathbf{r}, t)$ and $v_{2}(\mathbf{r}, t)$ be two external potentials that differ by more than an $\mathbf{r}$-independent function $C(t)$, and let $n_{1}(\mathbf{r}, t)$ and $n_{2}(\mathbf{r}, t)$ be the corresponding induced density fluctuations. Obviously, from Eq. (10) we have, for small $t-t_{0}$,

$$
n_{1}(\mathbf{r}, t)-n_{2}(\mathbf{r}, t) \sim(-1 / m) \nabla \cdot[\langle n(\mathbf{r})\rangle \nabla U(\mathbf{r}, t)],
$$

where

$$
U(\mathbf{r}, t)=\int_{t_{0}}^{t} d t^{\prime}\left(t-t^{\prime}\right)\left[v_{1}\left(\mathbf{r}, t^{\prime}\right)-v_{2}\left(\mathbf{r}, t^{\prime}\right)\right] .
$$

It is easy to prove by the differentiation of Eq. (11b) with respect to time that $\nabla U(\mathbf{r}, t)$ and $\nabla[\partial U(\mathbf{r}, t) / \partial t]$ cannot be identically zero at all times $t$ and all space points $\mathbf{r}$ when $v_{1}-v_{2}$ differs by more than an $\mathbf{r}$ independent function $C(t)$ over finite intervals of time $t^{\prime}$ at $t^{\prime}<t$. Therefore, following the treatment of Runge and Gross [see the discussion after Eq. (6) in Ref. 3 and also in Ref. 6], there must exist some finite time intervals over which $n_{1}(\mathbf{r}, t)-n_{2}(\mathbf{r}, t)$ is not identically zero when $\langle n(\mathbf{r})\rangle$ falls off rapidly enough to zero as $\mathbf{r} \rightarrow \infty$. (For a more rigorous statement about boundary conditions, see Ref. 6.) Thus there exists a one-to-one mapping between the external time-dependent potential $v(\mathbf{r}, t)$ and the induced density fluctuation $n(\mathbf{r}, t)$ in a small but finite time interval $t-t_{0}$ after the external potential is turned on if Eq. (8) is satisfied.

For external potentials which can be expanded into a Taylor series with respect to the time coordinate around $t=t_{0}$, it is easy to obtain the result of Runge and Gross [Eq. (6) of Ref. 3] by the differentiation of Eq. (3) with respect to time and the use of Eqs. (6) and (10).

Invertibility of the density-density response function $\chi\left(\mathbf{r}, \mathbf{r}^{\prime} ; \omega\right)$. - Now we consider the situation when a stationary system is perturbed by a weak external timedependent potential for a long period of time, so that one can consider the time Fourier transform of Eq. (3), i.e.,

$$
n(\mathbf{r}, \omega) \sim \int d^{3} r^{\prime} \chi\left(\mathbf{r}, \mathbf{r}^{\prime} ; \omega\right) v\left(\mathbf{r}^{\prime}, \omega\right)
$$

where $n(\mathbf{r}, \omega)$ and $v(\mathbf{r}, \omega)$ are the time Fourier transforms of the induced density fluctuation and the external potential, respectively. In this case, the problem of whether there exists a one-to-one mapping between $n(\mathbf{r}, t)$ and $v(\mathbf{r}, t)$ reduces to the problem of whether zeros exist in the mapping $n=\chi_{v}$, [i.e., Eq. (12)]. The problem has been studied by several authors, ${ }^{8,9}$ but no general theorem has been established, except in the case when the density distribution $n(\mathbf{r})$ of the stationary system varies slowly in space, so that a local-density approximation is applicable. ${ }^{9}$

We shall now prove the following theorem: For a system of fixed number of particles which satisfies the second law of thermodynamics, there is a one-to-one mapping between $n(r, \omega)$ and $v(r, \omega)$ (ii) if the system under consideration is at thermal equilibrium and (ii) if corresponding to this equilibrium state there is a unique minimum in the Helmoltz free energy $F$.

The proof of the above theorem proceeds as follows.

(i) Let $\chi^{\prime \prime}\left(\mathbf{r}, \mathbf{r}^{\prime} ; \omega\right)=\operatorname{Im} \chi\left(\mathbf{r}, \mathbf{r}^{\prime} ; \omega\right)$. First, we shall prove that if for all possible nonzero external potentials $v(\mathbf{r}, \omega)$ at given $\omega$,

$$
\int d^{3} r \int d^{3} r^{\prime} \omega v^{*}(\mathbf{r}, \omega) \chi^{\prime \prime}\left(\mathbf{r}, \mathbf{r}^{\prime} ; \omega\right) v\left(\mathbf{r}^{\prime}, \omega\right)>0,
$$

then there exists no zero in the mapping

$$
n(\mathbf{r}, \omega)=\int d^{3} r^{\prime} \chi\left(\mathbf{r}, \mathbf{r}^{\prime} ; \omega\right) v(\mathbf{r}, \omega) .
$$

The proof is by reductio ad absurdum: Assume that there exists an external potential $u(\mathbf{r}, \omega)$ such that

$$
\int d^{3} r^{\prime} \chi\left(\mathbf{r}, \mathbf{r}^{\prime} ; \omega\right) u\left(\mathbf{r}^{\prime}, \omega\right)=0
$$

Let $\quad \chi^{\prime}\left(\mathbf{r}, \mathbf{r}^{\prime} ; \omega\right)=\operatorname{Re} \chi\left(\mathbf{r}, \mathbf{r}^{\prime} ; \omega\right) \quad$ and $\quad u(\mathbf{r}, \omega)=u_{1}(\mathbf{r}, \omega)$ $+i u_{2}(\mathbf{r}, \omega)$ where $u_{1}$ and $u_{2}$ are real. Then from Eq. (14) we must have

$$
\chi^{\prime} u_{1}-\chi^{\prime \prime} u_{2}=0, \chi^{\prime} u_{2}+\chi^{\prime \prime} u_{1}=0,
$$


$\chi u$ being an abbreviation for $\int d r^{\prime} \chi\left(\mathbf{r}, \mathbf{r}^{\prime} ; \omega\right) u\left(\mathbf{r}^{\prime}, \omega\right)$. Therefore

$$
u_{2} \chi^{\prime} u_{1}=u_{2} \chi^{\prime \prime} u_{2}, \quad u_{1} \chi^{\prime} u_{2}+u_{1} \chi^{\prime \prime} u_{1}=0,
$$

where $u_{1} \chi u_{2}$ is an abbreviation for

$$
\int d^{3} r \int d^{3} r^{\prime} u_{1}(\mathbf{r}, \omega) \chi\left(\mathbf{r}, \mathbf{r}^{\prime} ; \omega\right) u_{2}\left(\mathbf{r}^{\prime}, \omega\right)
$$

etc. Since $\chi^{\prime}$ and $\chi^{\prime \prime}$ are Hermitian matrices, which fol- lows from the exact expression

$$
\chi\left(\mathbf{r}, t ; \mathbf{r}^{\prime}, t^{\prime}\right)=i\left\langle\left[n(\mathbf{r}, t), n\left(\mathbf{r}^{\prime}, t^{\prime}\right)\right]\right\rangle,
$$

we must have $u_{2} \chi^{\prime} u_{1}=u_{1} \chi^{\prime} u_{2}$ and therefore $u_{2} \chi^{\prime \prime} u_{2}$ $+u_{1} \chi^{\prime \prime} u_{1}=0$, which contradicts Eq. (13).

(ii) However, Eq. (13) is related to the work done by the external potential on the system which is given by ${ }^{7}$

$$
\Delta W=\int_{-\infty}^{+\infty} d t \int d^{3} r v(\mathbf{r}, t) \partial n(\mathbf{r}, t) / \partial t,
$$

which, with use of Eq. (3) and Fourier transformation in time, can be rewritten as ${ }^{7}$

$$
\Delta W=\int(d \omega / 2 \pi) \int d^{3} r \int d^{3} r^{\prime} \omega v^{*}(\mathbf{r}, \omega) \chi^{\prime \prime}\left(\mathbf{r}, \mathbf{r}^{\prime} ; \omega\right) v\left(\mathbf{r}^{\prime}, \omega\right)
$$

to second order in $v(\mathbf{r}, t)$.

For a stable system with a fixed number of particles in thermal equilibrium at fixed temperature $T, \Delta W \geq 0$ from thermodynamics. ${ }^{7,10}$ The equality sign holds only for reversible processes which are quasistatic ${ }^{10}$ (i.e., the $\omega=0$ processes). For the $\omega \neq 0$ processes, $\Delta W>0$ which implies from Eq. (19) that

$$
\int d^{3} r \int d^{3} r^{\prime} \omega v^{*}(\mathbf{r}, \omega) \chi^{\prime \prime}\left(\mathbf{r}, \mathbf{r}^{\prime} ; \omega\right)_{v}\left(\mathbf{r}^{\prime}, \omega\right)>0
$$

for all nonzero $\omega$ 's. Thus with (i) we conclude that there exists no zero in the mapping $n=\chi_{v}$ at finite $\omega$. In the static case, the invertibility of $\chi\left(\mathbf{r}, \mathbf{r}^{\prime} ; \omega=0\right)$ is also warranted by the Hohenberg-Kohn theorem (or Mermin's theorem at finite temperature ${ }^{11}$ ) which states that there exists a one-to-one mapping between the external potential $v(\mathbf{r})$ and the density distribution $n(\mathbf{r})$ when there is a unique minimum in the ground-state energy (or Helmoltz free energy at finite temperature). This completes the proof of our theorem.

Comments and conclusions. - In this Letter we have examined the possibility of formulating a time-dependent density-functional theory in the linear-response regime. We have proved that if Eq. (8) is valid, there exists a one-to-one mapping between an external time-dependent potential $v(\mathbf{r}, t)$ and the induced-density fluctuation $n(\mathbf{r}, t)$ in a small but finite time domain after the external potential is turned on. A sufficient condition for Eq. (8) to be valid is that all moments of the density-density response function are finite so that the expansion (6) is valid. However, this is probably not true in the case of a homogeneous interacting-electron gas because of a $\omega^{-11 / 2}$ tail $^{12}$ in the response function at large $\omega$ (this result has been obtained from the second-order perturbation theory ${ }^{12}$ ) and is, therefore, not expected to be true in the case of an inhomogeneous interacting-electron gas. However, the existence of the large-frequency tail simply implies that the response function is not expandable in integral powers of $t-t_{0}$ at small time. Let $p$ be the smallest nonintegral power of $t-t_{0}$ in a "valid" expansion of the response function $\chi$ at small time. If $p>1$, then Eq. (8) may still be valid and a one-to-one mapping between $v$ and $n$ may still be established. A basic as-

sumption that we have made in our analysis is the applicability of linear-response theory at small time $t-t_{0}$. It seems that this is valid as long as the system is changing continuously under the influence of the external potential around $t=t_{0}$.

We have also proved that there exists no zero in the mapping $n=\chi_{v}$ for a system in thermal equilibrium if, corresponding to this equilibrium state, there exists a unique minimum in the Helmoltz free energy. A basic assumption that we made in the proof is that the system under consideration obeys the second law of thermodynamics.

In summary, we have shown that it is possible to extend the work of Runge and Gross ${ }^{3}$ to the case of an arbitrary (finite) form of external potential and establish the existence of a one-to-one mapping between $v(\mathbf{r}, t)$ and $n(\mathbf{r}, t)$ in a finite time interval. Note that a one-toone mapping between $n$ and $v$ in a finite time interval is necessary for the establishment of a time-dependent density-functional theory. Our extension of the RungeGross result is valid only if Eq. (8) describes the shorttime behavior of the density-density response function correctly. The correctness of Eq. (8) is not proven in general cases in this Letter. Thus we feel that it is not clear at the present moment whether a time-dependent density-functional theory can be established in general. Precisely for this reason we turn to a more special case where the time-dependent potential is weak and we are interested in the long-time behavior of the system. This is the domain of experimental interest. We prove that there exists a one-to-one mapping between $n(\mathbf{r}, \omega)$ and $v(\mathrm{r}, \omega)$ under a very general thermodynamic condition and thus establish directly the possibility of formulating a density-functional theory in this regime. The question of $v$ representability is not being answered in either domain in this Letter. We have not attempted here to discuss various ways of implementing the theory in realistic calculations. Methods of constructing density functionals and performing calculations have been discussed elsewhere by several authors. ${ }^{3,5,8,13}$ 
(a) Present address: Department of Physics, Massachusetts Institute of Technology, Cambridge, MA 02139.

${ }^{1}$ P. Hohenberg and W. Kohn, Phys. Rev. 136, B864 (1964); see, also, W. Kohn and L. J. Sham, Phys. Rev. 140, A1133 (1965).

${ }^{2}$ For a review, see Theory of the Inhomogeneous Electron Gas, edited by S. Lundqvist and N. H. March (Plenum, New York, 1983).

${ }^{3}$ E. Runge and E. K. U. Gross, Phys. Rev. Lett. 52, 997 (1984).

${ }^{4}$ T. Li and P. Tong, Phys. Rev. A 31, 1950 (1985).

${ }^{5}$ K. Kohl and R. M. Dreizler, Phys. Rev. Lett. 56, 1993 (1986).

${ }^{6}$ A. K. Dhara and S. K. Ghosh, Phys. Rev. A 35, 442 (1987).

${ }^{7}$ See, for example, D. Forster, Hydrodynamic Fluctuations, Broken Symmetry, and Correlation Functions (Benjamin,
New York, 1983).

${ }^{8}$ V. Peuckert, J. Phys. C 11, 4945 (1978).

${ }^{9}$ S. Chakravarty, M. B. Fogel, and W. Kohn, Phys. Rev. Lett. 43, 775 (1979).

${ }^{10}$ See, for example, P. Glansdorff and I. Prigogine, Thermodynamics of Structure, Stability, and Fluctuations (WileyInterscience, New York, 1971).

${ }^{11}$ N. D. Mermin, Phys. Rev. 137, A1441 (1965).

${ }^{12}$ A. J. Glick and W. F. Long, Phys. Rev. B 4, 3455 (1971); see, also, E. K. U. Gross and W. Kohn, Phys. Rev. Lett. 55, 2850 (1985).

${ }^{13}$ E. K. U. Gross and R. M. Dreizler, in Density Functional Methods in Physics, edited by R. M. Dreizler and J. da Providencia, NATO Advanced Study Institute Series B, Physics, Vol. 123 (Plenum, New York, 1985), p. 81. 